
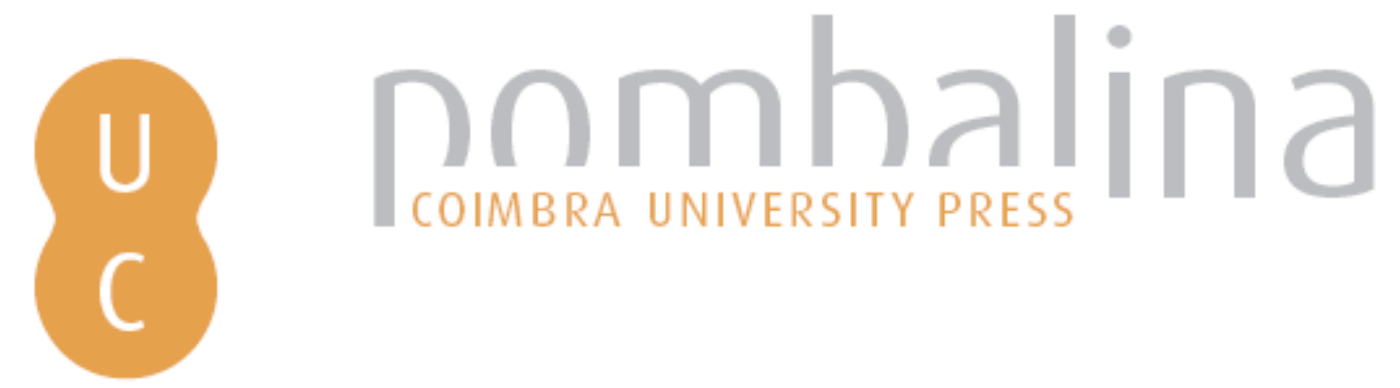

\title{
Una vera fashion consciousness: o italiano da moda e o empréstimo linguístico: breves notas a um artigo de VanityFair.it
}
Autor(es):
Sismondini, Alberto
Publicado por: Imprensa da Universidade de Coimbra
URL
persistente:
URI:http://hdl.handle.net/10316.2/40002
DOI:
DOI:https://doi.org/10.14195/978-989-26-1115-0_10
Accessed : $\quad$ 26-Apr-2023 14:43:49

A navegação consulta e descarregamento dos títulos inseridos nas Bibliotecas Digitais UC Digitalis, UC Pombalina e UC Impactum, pressupõem a aceitação plena e sem reservas dos Termos e Condições de Uso destas Bibliotecas Digitais, disponíveis em https://digitalis.uc.pt/pt-pt/termos.

Conforme exposto nos referidos Termos e Condições de Uso, o descarregamento de títulos de acesso restrito requer uma licença válida de autorização devendo o utilizador aceder ao(s) documento(s) a partir de um endereço de IP da instituição detentora da supramencionada licença.

Ao utilizador é apenas permitido o descarregamento para uso pessoal, pelo que o emprego do(s) título(s) descarregado(s) para outro fim, designadamente comercial, carece de autorização do respetivo autor ou editor da obra.

Na medida em que todas as obras da UC Digitalis se encontram protegidas pelo Código do Direito de Autor e Direitos Conexos e demais legislação aplicável, toda a cópia, parcial ou total, deste documento, nos casos em que é legalmente admitida, deverá conter ou fazer-se acompanhar por este aviso.

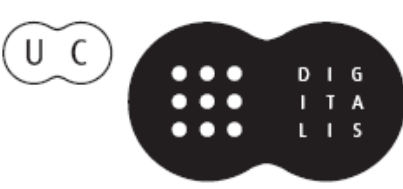




\section{JOÃO CORRÊA-CARDOSO MARIA DO CÉU FIALHO}

(Coordenadores)

\section{A LINGUAGEM NA PÓLIS}

IMPRENSA DA UNIVERSIDADE DE COIMBRA COIMBRA UNIVERSITY PRESS
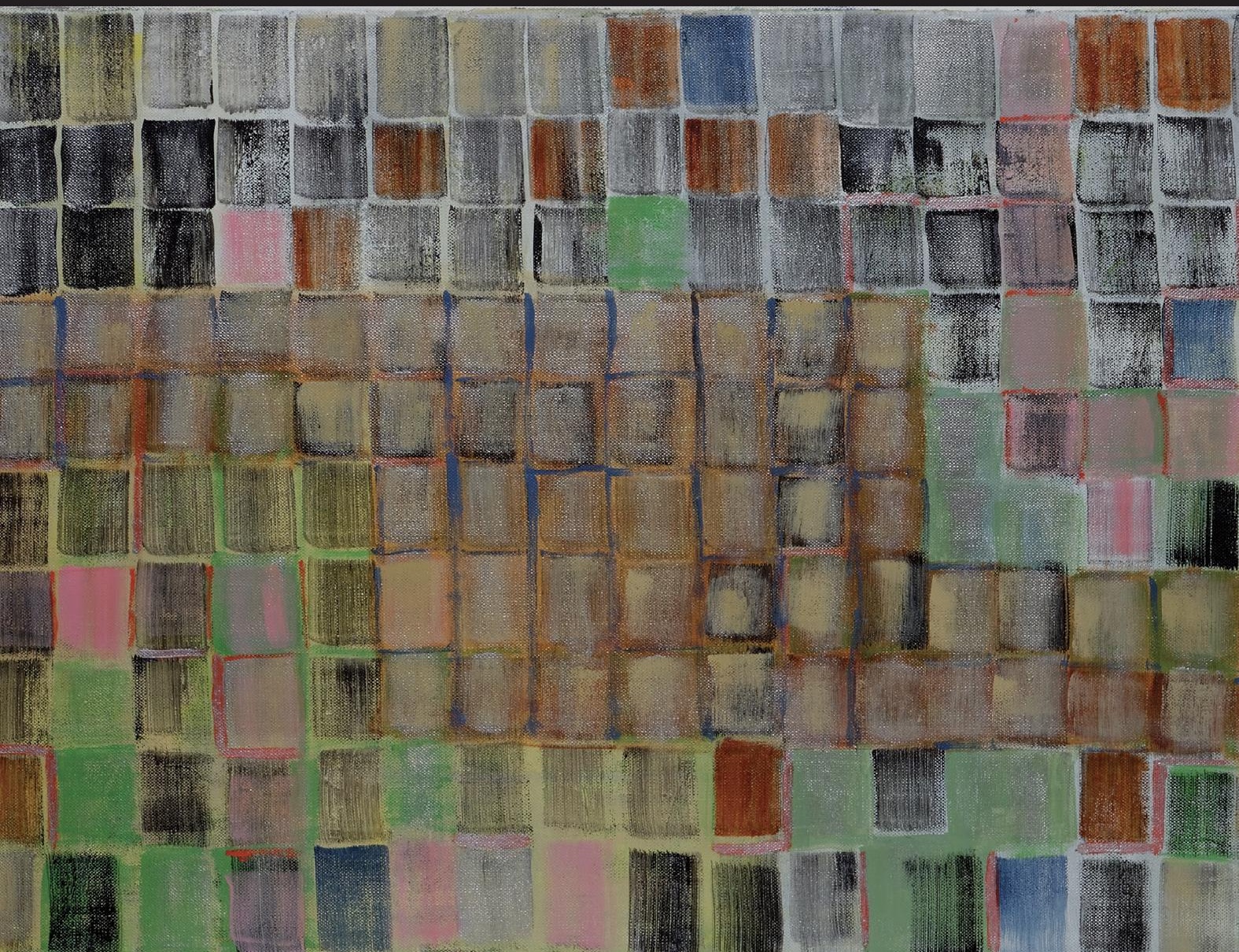


\section{UNA VERA FASHION CONSCIOUSNESS. O ITALIANO DA MODA E O EMPRÉSTIMO L I N G U Í S T I C O : BREVES NOTAS A UM ARTIGO DE VANITYFAIR.IT}

Alberto Sismondini Universidade de Coimbra sarvagi@fl.uc.pt

Resumo: Partindo das considerações de Roland Barthes a respeito do universo da moda, este texto pretende focar o fenómeno do empréstimo linguístico no léxico italiano da moda, conforme a análise de um artigo publicado pela edição italiana da revista Vanity Fair, pertencente ao grupo norte-americano Hearst. Uma análise dos lexemas estrangeiros utilizados e o seu valor percentual na totalidade dos termos publicados, permite observar a mutação de tendência ocorrida nas últimas décadas, em que a língua inglesa substiuiu o francês como "língua franca" da moda nas publicações italianas. Palavras chave: Roland Barthes, moda, empréstimo linguístico, italiano, inglês Abstract: Starting from Roland Barthes' point of view on the fashion universe, this work aims to focus on the linguistic loan as a phenomenon in Italian fashion current lexicon. The results are obtained from an article published in the Italian edition of Vanity Fair, actually belonging to the U.S. Hearst group. Through this brief survey, which points out foreign lexemes and their strength over the whole amount of words published in the text, a growing tendency of the last decades is, once more, revealed: English is taking the place of French as "lingua franca» in Italian fashion publications. Keywords: Roland Barthes, fashion, linguistic loan, Italian, English 
Há poucas semanas recebi de uma amiga, docente de estudos clássicos e de literatura italiana no conceituado liceu da minha cidade natal, um mail e um perentório convite ("devi assolutamente leggerlo!», dizia o texto) para ler um artigo divulgado em rede, consagrado à vida de mulheres de poder, da versão italiana da revista internacional Vanity Fair.

Mesmo suspeitando houver algum engano, de não ser eu o destinatário, abri o hipertexto e percebi que de facto, mais do que a admirar as imagens de primeiras damas e aprendizes rainhas, o convite dizia respeito à análise das notas que concisamente acompanhavam a série de 29 slides, a compor a apresentação do artigo, cujas 987 palavras seguem reproduzidas. É suficiente uma rápida vista de olhos para deparar com a forte incidência de palavras estrangeiras em uso no léxico italiano contemporâneo das revistas de moda.

\section{Donne di potere. E di stile ${ }^{1}$ \\ Da Michelle Obama a Kate Middleton passando per Cristina Kirchner: sotto la lente, il look di first lady, presidenti e regine}

di R. Fiori 1.04.2011

"Michelle dresses to win" (Michelle Obama si veste per vincere) aveva titolato il New York Times, il 6 novembre 2008, ovvero il giorno dopo la vittoria delle presidenziali americane da parte del marito Barack. Quel giorno, la neo first lady veniva incoronata anche fashion icon. In quell'occasione Michelle O', come era stata ribattezzata in memoria di Jackie Kennedy, aveva indossato un (criticatissimo) abito giallo lime della stilista americano-cubana Isabel Toledo; per il gala di insediamento alla Casa Bianca un abito bianco (che sembrava da sposa) di Jason Wu. Uno stile

\footnotetext{
${ }^{1}$ http://www.vanityfair.it/starstyle/i-like/2011/03/28/le-fashion-icon-della-politica
} 
estremamente personale il suo, guidato unicamente dalla tempra del suo carattere.» ( p.2)

«Mrs. Obama tiene a debita distanza il fashionbiz: non flirta più di tanto con le maison, anzi, si diverte a mostrare la propria indipendenza critica mixando marchi low budget e alta moda. Con Michelle la moda riscopre la sua (spesso dimenticata) funzione di metalinguaggio. La first lady americana è consapevole del potere della moda e attraverso le sue scelte di stile veicola le sue scelte etiche come quella di sostenere stilisti emergenti trapiantati negli States (vedi Thakoon Panichgul, Jason Wu e Maria Pinto). Alcune linee guida del suo guardaroba: abiti smanicati (per mettere in mostra le belle braccia ancora toniche), le scarpe ultra flat (per non superare il marito) e colori segnaletici (per emergere dall'ombra del consorte nonché presidente degli States).»(p.3)

«Dopo anni di passerelle e copertine patinate, la ex top model Carla Bruni non ha bisogno di consigli in fatto di stile. La prèmiere dame francese con un passato da modella, cantante, attrice, si destreggia, infatti, con innata disinvoltura e consapevolezza tra marchi, tagli e icone di riferimento (lei ha scelto Jaqueline Kennedy). Dalla sua, il fisico perfetto che, però, sembra voler nascondere per acquistare una certa credibilità come donna di potere.» ( p.8)

"Monocromia, lunghezze midi e scarpe flat (l'altezza del marito Nikolas Sarkozy non dà molta scelta) sono i capisaldi del suo guardaroba, elegante, ma non troppo originale. Che sia una strategia per non offuscare monsieur le President o è semplicemente il suo modo di interpretare il ruolo-personaggio di first lady? Il guardaroba di Carlà trabocca di griffatissimi tailleur taylor made, petit noir, cappellini e guanti che ci trasportano lontano nel tempo, quando ancora ogni capo aveva la sua occasione.» ( p.9)

"Da quando è passata dallo status di fidanzata storica a quello di fidanzata ufficiale del principe William d'Inghilterra, Kate 
Middleton è diventata la fashion guru delle suddite inglesi. Kate sa che il suo look è sotto la lente d'ingrandimento di fan e riviste ed è forse per questo che per il momento sembra andarci cauta. Lo stile della futura principessa di Cambridge sembra essere, infatti, ancora in fase embrionale: orli al ginocchio, abiti monocromi e cappellini secondo tradizione segnano il suo ingresso - quasi nell'anonimato in fatto di stile - a Bunkingham Palace. Come lei, anche una giovanissima Lady Diana, trentuno anni fa, entrò nel palazzo reale inglese senza una vera fashion consciousness, un po' per via del suo carattere riservato, un po' per la rigida etichetta inglese. Con gli anni, sviluppò un preciso gusto stilistico insieme al senso di ribellione alla corte. Kate, da par sua, ha, però, già dimostrato di avere un penchant per le balze e - per non scontentare forse il made in Uk - per il tweed.» (p.13)

"Christina Kirchner è passata da first lady, al fianco del marito Néstor, a prima donna Presidente dell'Argentina. In corsa per il secondo mandato presidenziale, Cristina è una donna di polso, ma estremamente femminile. Il suo punto debole: le scarpe con plateau. Per via della sua passione per le scarpe, la Prima Signora è stata soprannominata Imelda Marcos, come la moglie del ex dittatore filippino. Atout del suo guardarobe i robe-manteau di broccato, tailleur gonna o pantalone di foggia sartoriale e i mini bolero portati sopra i tubini per creare uno strategico gioco di proporzioni. La palette colori vede protagonista su tutti il bianco che fa risaltare il suo incarnato super tan (tutto l'anno). La sua firma di stile? L'accostamento di tessuti shine e mat: camicia di raso e gonna di lana, abiti effetto jaquard sotto il cappotto lucido e viceversa.» ( p. 18)

"Ad avere gli occhi e i flash puntati contro Letizia Ortiz era già abituata dai tempi del telegiornale alla Cnn. Il suo gusto ha avuto tutto il tempo di affinarsi anche se il passaggio da anchorwoman a consorte del principe Felipe di Spagna non è stato facile. Nel 
giro di pochi anni, Letizia è, infatti, dimagrita moltissimo, tanto da insinuare il sospetto di anoressia. Dal tailleur rigoroso da mezzobusto televisivo, la neo principessa delle Asturie è passata a coloratissimi tubini che sottolineano la silhouette sottile (anche immediatamente dopo le due gravidanze). Conscia di essere sempre sotto l'occhio attento dei sudditi Letizia sembra non avere mai un'aria davvero rilassata. Anche il casual wear è studiato nei minimi dettagli: è informale ma griffatissimo e soprattutto coordinato. Il suo è un mix calcolato di buon gusto e consapevolezza del ruolo. La sua fortuna? Avere un marito altissimo che le permette di poter alternare in tutta libertà décolletée altissime - le sue preferite- e ballerine ultra flat.» (p.22)

"Regalità e stile innati, la regina Rania di Giordania sa valorizzare la propria figura, perfetta nonostante le quattro gravidanze, e accostare con gusto colori e accessori. Corteggiatissima dagli stilisti internazionali, Rania sa scegliere, a seconda dell'occasione, il capo giusto nel rispetto della cultura del suo Paese, ma senza risultare bigotta. Per le occasioni internazionali Rania opta per abiti dal taglio minimal non troppo aderenti al corpo e con l'orlo dal ginocchio in giù. Per le serate di gala "in casa", invece, la consorte del re Abdhulla si lascia sedurre dai tessuti preziosi, dai ramage floreali, dalle applicazioni bijoux e dai drappeggi.»(p.26)

Procurando sistematizar a série de lexemas estrangeiros presentes no texto, fui conferir, em dois dicionários italianos monolingues em rede, de comprovada qualidade, ${ }^{2}$ se estes empréstimos não integrados já constavam das listas de palavras consagradas no vocabulário transalpino.

${ }^{2}$ Grande dizionario italiano de Aldo Gabrielli in http://dizionari.hoepli.it/ Dizionario_Italiano.aspx?idD=1, Milano, Hoepli e Dizionario Italiano Sabatini e Coletti de Francesco Sabatini e Vittorio Coletti, in http://dizionari.corriere.it/dizionario_italiano/index.shtml Firenze, Giunti. 
Anglicismos

(49 palavras, 4,96\% do total):

\begin{tabular}{|c|c|c|c|c|}
\hline Página & Palavra / locução & Dic. A & Dic. B & Tradução italiana \\
\hline 2 & first lady & Sim & Sim & La moglie del presidente \\
\hline 2 & fashion icon & Não* & Não* & icona della moda \\
\hline 2 & lime & Sim & Sim & lime \\
\hline 3 & Mrs. [Obama] & Não & Não & la signora [Obama] \\
\hline 3 & fashionbiz & Não* & Não* & $\begin{array}{l}\text { il mondo degli affari nel campo della } \\
\text { moda }\end{array}$ \\
\hline 3 & mixando & Não & Não & mescolando \\
\hline 3 & low budget & Não & Não & a buon prezzo / economici \\
\hline 3 & first lady & Sim & Sim & la moglie del presidente \\
\hline 3 & States & Não & Não & gli Stati Uniti d'America \\
\hline 3 & ultra flat & Não & Não & ballerine \\
\hline 8 & top model & Sim & Sim & indossatrice di grande successo \\
\hline 9 & flat & Não & Não & ballerine \\
\hline 9 & first lady & Sim & Sim & la moglie del presidente \\
\hline 9 & taylor made & Não & Não & sartoriale \\
\hline 13 & fashion guru & Não* & Não* & guru della moda \\
\hline 13 & look & Sim & Sim & immagine, aspetto \\
\hline 13 & fan & Sim & Sim & sostenitore, ammiratore \\
\hline 13 & $\begin{array}{l}\text { fashion } \\
\text { coinsciousness }\end{array}$ & Não* & Não* & $\begin{array}{l}\text { consapevolezza dei propri gusti nella } \\
\text { moda }\end{array}$ \\
\hline 13 & penchant & Não & Não & avere un debole \\
\hline 13 & made in UK & Não & Não & prodotti britannici \\
\hline 13 & tweed & Sim & Sim & tweed, tessuto in lana \\
\hline 18 & first lady & Sim & Sim & la moglie del presidente \\
\hline 18 & super tan & Não & Não & molto abbronzato \\
\hline 18 & shine e mat & Não & Não & lucido e opaco \\
\hline 22 & flash & Sim & Sim & lampo \\
\hline 22 & anchorwoman & $\operatorname{Sim}^{* *}$ & Sim & conduttrice di programmi. \\
\hline 22 & casual wear & $\operatorname{Sim}^{* * * *}$ & Sim*冰冰 & Abbigliamento informale / sportivo \\
\hline 22 & mix & Sim & Sim & combinazione \\
\hline 22 & ultra flat & Não & Não & ballerine \\
\hline 26 & minimal & Não & Não & minimale \\
\hline \multicolumn{2}{|l|}{ NOTAS: } & \multicolumn{3}{|c|}{ * existe a palavra "fashion" } \\
\hline \multicolumn{2}{|c|}{$\begin{array}{l}\text { *** existe apenas o termo } \\
\text { masculino "anchorman" }\end{array}$} & \multicolumn{3}{|c|}{ ***** existe apenas o termo "casual" } \\
\hline
\end{tabular}


Galicismos:

(20 palavras; $2,03 \%$ do total)

\begin{tabular}{|l|l|l|l|l|}
\hline Página & Palavra / locução & Dic. A & Dic. B & Tradução italiana \\
\hline 2 & gala & Sim & Sim & Festa, raduno, evento \\
\hline 3 & maison & Não & Não & casa di moda \\
\hline 8 & première dame & Não & Não & la moglie del presidente \\
\hline 9 & Monsieur le président & Não & Não & Signor Presidente \\
\hline 9 & tailleur & Sim & Sim & tailleur \\
\hline 9 & Petit noir & Não & Não & $\begin{array}{l}\text { abito da cocktail trasformato in } \\
\text { tenuta serale }\end{array}$ \\
\hline 18 & plateau & Não & Não & zeppa, suola rialzata \\
\hline 18 & Atout & Sim & Sim & opportunità \\
\hline 18 & robe-manteau & Sim & Não & $\begin{array}{l}\text { abito femminile di foggia simile a } \\
\text { cappotto }\end{array}$ \\
\hline 18 & palette & Não & Não & gamma di colori \\
\hline 18 & jacquard & Sim & Sim & jacquard \\
\hline 22 & tailleur & Sim & Sim & tailleur \\
\hline 22 & silhouette & Sim & Sim & figura \\
\hline 22 & décolletée & Sim & Sim & scarpe scoperte \\
\hline 26 & ramages & Sim & Não & motivo floreale su stoffa \\
\hline 26 & bijou & Sim & Sim & gioiello, ninnolo \\
\hline
\end{tabular}

Um primeiro resultado demonstra como das 49 palavras inglesas apresentadas, 30 não estão presentes nos dois dicionários, perfazendo uma média de $61,2 \%$. De igual modo, das 20 palavras francesas contempladas, 10 não constam das mesmas fontes (é uma média de $50 \%$, portanto, mais baixa do que a anteriormente indicada).

Considerando uma base de 250.000 lexemas, ${ }^{3}$ Tullio De Mauro afirma que os estrangeirismos presentes no seu Grande dizionario italiano dell'uso (1999), entre os que já foram integrados na morfologia da língua hóspede e os de importação direta, o primado vai para os helenismos (8354 - a perfazer uma percentagem do 3,34\%), seguidos pelos anglicismos (5510 - 2, 2\%) e pelos lexemas vindos do francês (4370 - 1,75\%). No texto analisado, os empréstimos não integrados chegam até $7 \%$ da totalidade de palavras, revestindo

3 Tullio de Mauro (2005), La fabbrica delle parole, Torino, Utet, p. 136. 
um valor primordial na economia semântica da escrita, tornando-a "geradora" de novas relações entre significantes e significado, às vezes sem um termo correspondente em italiano: veja-se o caso de "fashionbiz" ou de "petit-noir». Observemos também que o tema do artigo, a vida de personalidades famosas e o seu relacionamento com a indumentária em eventos oficiais, obriga o autor a utilizar uma linguagem pensada para a fruição de um número avultado de leitores, uma língua geral, de sintaxe linear, "abrilhantada" porém pelo uso de termos importados, quase a criar uma forma de gíria, caindo no abuso de negligenciar palavras italianas existentes e perfeitamente aptas a ser usadas em seu lugar. ${ }^{4}$ Note-se a intenção de aproveitar um léxico exótico ou especializado para criar um sentido de fascinação nos leitores.

No que diz respeito à literatura científica italiana, valem as considerações de Berruto 5 sobre as variedades diafásicas caracterizadas por um léxico especial, em relação a determinados domínios extra-linguísticos e às correspondentes áreas de significados. Estas reflexões são utilizadas por Alberto Sobrero ${ }^{6}$ para postular uma definição geral de "lingue speciali» [línguas especiais] para as variações das línguas ligadas a setores e disciplinas peculiares, tais como a medicina, a física e o desporto. Continuando na sua classificação este autor passa a definir "lingue specialistiche» [línguas especializadas] as típicas de disciplinas com alto grau de especialização. Interpretando o texto de Sanger, Dungworth e McDonald (1980), ${ }^{7}$

\footnotetext{
${ }^{4}$ Cf. Federica Pellegrino, Forestierismi e latinismi, in Semplificazione del linguaggio amministrativo - Manuale di stile no site http://www.maldura.unipd.it/buro/ gel/gel12.html (15.04.2012).

5 Gaetano Berruto (1997) "Varietà diamesiche, diastratiche, diafasiche" in Alberto Sobrero (org.) Introduzione all'italiano contemporaneo - La variazione e gli usi, Bari, Laterza, pp. 37-92.

${ }^{6}$ Alberto A. Sobrero, (1997) "Lingue speciali" in Sobrero, op.cit., pp. 237-277.

7 Sager, Juan C.- Dungworth, David- McDonald, Peter F. (1980) English special languages. Principles and practice in science and technology. Wiesbaden, Oscar Brandstetter Verlag.
} 
Sobrero define a língua especializada como um subsistema ou extensão da língua especial, que partilha o mesmo sistema gramatical adicionado de um léxico próprio; a sua sintática é também redesenhada para atingir um público-alvo representado por iniciados na matéria, segundo critérios formais de economia, de objetividade e de propriedade. ${ }^{8}$ Continuando na sua análise, Sobrero observa que os textos especializados neutralizam qualquer aspeto ligado às funções emotiva, expressiva e fática. Estes textos têm características discursivas peculiares tal como descrições, definições e comparações. O autor insiste em indicar o léxico como zona de fronteira entre a língua especializada e uma língua especial mais genérica (língua setorial). Outros fenómenos morfossintáticos relevantes consubstanciam-se na nominalização, na perda de valor do verbo, na alta densidade semântica, no uso reduzido das orações subordinadas, na utilização da forma passiva e dos modos impessoais, além dos já citados neologismos, empréstimos não integrados e acrónimos. ${ }^{9}$

A linguagem setorial, como Sobrero indica, usa a língua geral para não perder contacto com o seu público definido, generalista, efetuando pontualmente algumas incursões na linguagem especializada, principalmente para apropriações lexicais, captando termos usados por iniciados.

Um estudo de Massimo Cavaliere, ${ }^{10}$ na senda desta reflexão, elaborou um esquema a considerar a tipologia de público das revistas de moda e o tipo de linguagem utilizadas:

\footnotetext{
8 Alberto Sobrero, ibidem.

9 Sobrero (1997), op. cit. pp. 243-251.

10 Massimo Cavaliere (2006) Il linguaggio della moda tra parole e immagini, Tesi Università degli Studi "Aldo Moro", Bari; uma súmula da tese em http://www. tesionline.it/consult/preview.jsp?pag=1\&idt=15575 (16.04.2012).
} 


\begin{tabular}{|l|l|}
\hline Tipologia de leitores & Caraterísticas morfossintáticas e lexicais das revistas \\
\hline Profissionais da moda & $\begin{array}{l}\text { Língua especializada (técnico-económica) } \\
\text { nominalização } \\
\text { estrangeirismos } \\
\text { justaposições nominais } \\
\text { acrónimos }\end{array}$ \\
\hline $\begin{array}{l}\text { Atores e consumidores de } \\
\text { moda }\end{array}$ & $\begin{array}{l}\text { Língua setorial } \\
\text { uso de wordplays } \\
\text { estrangeirismos } \\
\text { Jargão cool } \\
\text { decalques semânticos } \\
\text { amálgamas }\end{array}$ \\
\hline Massas & $\begin{array}{l}\text { Língua popular } \\
\text { sintaxe linear } \\
\text { polissemia } \\
\text { glosas explicativas }\end{array}$ \\
\hline
\end{tabular}

A leitura do artigo de Vanityfair.it, por razões acima mencionadas, sugere a inscrição do texto na categoria medial, sendo o público alvo parte da fashionable people, fundamental para o sucesso comercial da moda.

O mesmo estudo evidencia quanto o mundo editorial italiano deste setor está dependente dos Estados Unidos por razões económicas, sendo o mercado americano um dos mais importantes para as exportações das marcas italianas. O autor também realça a mutação de estilo que a revista Vogue America imprimiu a este domínio nos últimos vinte anos, sob a orientação da célebre diretora Anna Wintour.

Além das revistas, alguns romances emblemáticos, inspiradores de filmes ou de séries tais como The Devil Wears Prada, Sex and the City ou Desperate Housewives alcançaram uma audiência não necessariamente vincada no campo da moda e desenvolveram um papel primordial na evolução do gosto de um público à escala planetária. Esta audiência tornou-se potencial cliente de vários produtos do "luxo globalizado", a título de exemplo, as sandálias do estilista Manolo Blahnik. 
Roland Barthes ${ }^{11}$ defendia que, passando pela escrita, a moda se tornava narrativa e as revistas do setor "geradoras de sentido" do imaginário coletivo. Após décadas da publicação do Sistema da moda, é interessante observar a função contínua de certos lexemas ao tornarem-se «divindades naturais produtoras do vestuário».12 Já no original do autor gaulês, duas das palavras citadas eram constituídas por empréstimos não integrados ("cocktail» e "week-end»). Agora estes lexemas também pertencem legitimamente ao léxico italiano e são substituídos por outros mais inovadores («ultra flat» ou «shine e mat", por exemplo) que evocam, nos seus enunciadores e recetores, informações que ultrapassam a mera semântica da tradução literal do termo.

No italiano contemporâneo - uma língua não sujeita a políticas de protecionismo linguístico pelo governo, tal como o francês assiste-se ao avanço de terminologia estrangeira, nomeadamente no domínio permeável da moda e da publicidade, desde sempre sensíveis à atração de novos pólos internacionais da criatividade.

$\mathrm{Na}$ atualidade, é de facto a língua inglesa dos Estados Unidos que exerce o maior poder de influência sobre a língua setorial da moda na pátria de Dante.

\section{Bibliografia}

Barthes, Roland (1967), Le Système de la mode, Paris, Seuil. (1981), O Sistema da moda, Lisboa, Edições 70.

Berruto, Gaetano (1997), Varietà diamesiche, diastratiche, diafasiche. In: Alberto A. Sobrero (org.) Introduzione all'italiano contemporaneo - La variazione e gli usi, Bari, Laterza.

Cavaliere, Massimo (2006), Il linguaggio della moda tra parole e immagini, Tesi Università degli Studi “Aldo Moro”, Bari.

\footnotetext{
11 Roland Barthes (1967) Le Système de la mode, Paris, Seuil.

12 Roland Barthes (1981) O Sistema da moda, Lisboa, Edições 70, p. 310.
} 
de Mauro, Tullio (2005), La fabbrica delle parole, Torino, Utet.

Gabrielli, Aldo, Grande dizionario italiano. In: http://dizionari.hoepli.it/Dizionario_ Italiano.aspx?idD=1, Milano, Hoepli

http://www.vanityfair.it/starstyle/i-like/2011/03/28/le-fashion-icon-della-politica

Pellegrino, Federica (2001), Forestierismi e latinismi. In: Semplificazione del linguaggio amministrativo - Manuale di stile no site http://www.maldura.unipd.it/buro/ gel/gel12.html (15.04.2012)

Sabatini, Francesco / Coletti, Vittorio, Dizionario Italiano. In: http://dizionari. corriere.it/dizionario_italiano/index.shtml Firenze, Giunti.

Sager, Juan C./ Dungworth, David / McDonald, Peter F. (1980), English special languages. Principles and practice in science and technology. Wiesbaden, Oscar Brandstetter Verlag.

Sobrero, Alberto A. (1997) Lingue speciali. In: Alberto A. Sobrero (org.) Introduzione allitaliano contemporaneo - La variazione e gli usi, Bari, Laterza, pp.237-277. 